\title{
REMARKS ON FINITE GROUPS DEFINED BY GENERATING RELATIONS
}

\author{
ROBERT FRUCHT
}

1. Introduction. After establishing a duplication principle (\$2) which enables us to derive a group of order $2 h$ with $k+1$ involutory generators from any group of order $h$ with $k$ generators, we shall prove the following combination principle:

Let $\mathfrak{F}$ and $(5)$ be two groups, each having $k$ generators, such that the $r$ th

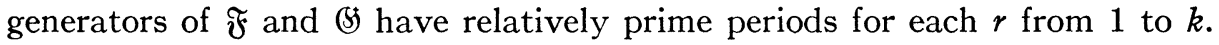
Then the direct product $\mathfrak{F} \times(\xi)$ can likewise be generated by $k$ elements (instead of $2 k$, as might be necessary without the condition imposed on the periods).

In the rest of the paper these two theorems are used as tools in the topological problem of finding symmetrical graphs of degree 3 . Indeed, by a method that essentially goes back to Cayley and has already been used by the author in an earlier paper (6), any group with three involutory generators gives rise to a graph of degree 3, and the "duplication principle" enables us to derive such a group from any group $\mathfrak{S}$ with two generators. The corresponding graph has $2 n$ vertices, where $n$ is the order of $\mathfrak{S}$, but it will be symmetrical only if $\mathfrak{S}$ satisfies a certain symmetry condition ( $\$ 4$ ). Some such groups can be combined by the "combination principle" to yield interesting examples of symmetrical graphs $(\$ \$ 5,6)$.

I wish to take this opportunity to thank H. S. M. Coxeter for his criticism of the present paper in manuscript, and especially for adding some of the examples, and to thank R. M. Foster for telling me about the symmetrical graphs found by him.

2. The duplication principle. Let $\mathfrak{S}$ denote a finite abstract group whose generators $S_{1}, S_{2}, \ldots, S_{k}$ are subject to the sole defining relations

$$
f_{i}\left(S_{1}, S_{2}, \ldots, S_{k}\right)=1 \quad(i=1,2, \ldots, m),
$$

where $f_{i}\left(S_{1}, S_{2}, \ldots, S_{k}\right)$ denotes a product of powers of some or all of the generators. Let (5) denote an abstract group whose generators $T_{1}, T_{2}, \ldots, T_{k+1}$ are subject to the sole defining relations

$$
\begin{aligned}
& T_{1}{ }^{2}=T_{2}{ }^{2}=\ldots=T_{k+1}{ }^{2}=1, \\
& f_{i}\left(T_{1} T_{2}, T_{2} T_{3}, \ldots, T_{k} T_{k+1}\right)=1 \quad(i=1,2, \ldots, m) .
\end{aligned}
$$

Then the order of $(5)$ is twice that of $\mathfrak{S}$.

Received April 10, 1954. 
Proof. The mapping

$$
T_{1} T_{2} \rightarrow S_{1}, \quad T_{2} T_{3} \rightarrow S_{2}, \ldots, T_{k} T_{k+1} \rightarrow S_{k}
$$

establishes an isomorphism between $\mathfrak{S}$ and a subgroup

$$
\left\{T_{1} T_{2}, T_{2} T_{3}, \ldots, T_{k} T_{k+1}\right\}
$$

of (5. This subgroup is of index 2 since it consists of all those elements of (5) which are products of even numbers of $T^{\prime}$ s, e.g.,

$$
T_{3} T_{1}=T_{3} T_{2} \cdot T_{2} T_{1}=\left(T_{2} T_{3}\right)^{-1}\left(T_{1} T_{2}\right)^{-1} .
$$

Example. Applying this principle to the cyclic group $\mathfrak{S}_{n}$, defined by

$$
S^{n}=1 \text {, }
$$

we obtain the dihedral group $\mathfrak{D}_{2 n}$ (of order $2 n$ ) in the form

$$
T_{1}^{2}=T_{2}^{2}=\left(T_{1} T_{2}\right)^{n}=1 \text {. }
$$

When $n=2$, this is the four-group $\mathfrak{\Im}_{2} \times \mathfrak{E}_{2}$. (Thus $(5)$ is sometimes, but not always, the direct product of $\mathfrak{S}$ and the group of order 2.)

More generally, whenever $\mathfrak{S}$ is Abelian and $k=1$ or 2 , $(5)$ is "generalized dihedral" in the sense of Miller (9, p. 168).

Remark. It is sometimes convenient to give $\mathfrak{S}$ an extra generator $S_{k+1}$, corresponding to $T_{k+1} T_{1}$, and to insert the extra relation

(10, p. 26).

$$
S_{1} S_{2} \ldots S_{k} S_{k+1}=1
$$

3. The combination principle. Let $\mathfrak{F}$ denote a finite group whose generators $R_{r}(r=1,2, \ldots, k)$ are subject to the sole defining relations

$$
f_{i}\left(R_{1}, R_{2}, \ldots, R_{k}\right)=1 \quad(i=1,2, \ldots, m) .
$$

Let (\$) denote another group having the same number of generators $S_{1}, S_{2}, \ldots$, $S_{k}$, subject to the relations

$$
g_{i}\left(S_{1}, S_{2}, \ldots, S_{k}\right)=1 \quad(i=1,2, \ldots, n) .
$$

Let $a_{r}$ be the period of $R_{r}$ (in $\mathfrak{F}$ ), and $b_{r}$ that of $S_{r}$ (in (\$) ). The direct product $\mathfrak{F} \times$ \& can obviously be generated by $2 k$ elements. If, for each $r, a_{r}$ and $b_{r}$ are relatively prime, $\mathfrak{F} \times$ (5) is isomorphic with the group $\Re$ having only $k$ generators, say $U_{r}(r=1,2, \ldots, k)$, subject to the defining relations

$$
\begin{aligned}
& f_{i}\left(U_{1}^{b_{1}}, U_{2}^{b_{2}}, \ldots, U_{k}^{b_{k}}\right)=1 \\
& (i=1,2, \ldots, m), \\
& g_{i}\left(U_{1}^{a_{1}}, U_{2}^{a_{2}}, \ldots, U_{k}^{a_{k}}\right)=1 \\
& (i=1,2, \ldots, n) \text {, } \\
& U_{r}^{a_{r}} U_{s}^{b_{s}}=U_{s}^{b_{s}} U_{r}^{a_{r}} \\
& (r \neq s ; r, s=1,2, \ldots, k) \text {. }
\end{aligned}
$$


Proof. $\Re$ contains a subgroup

$$
\mathfrak{R}_{F}=\left\{U_{1}^{b_{1}}, U_{2}^{b_{*}}, \ldots, U_{k}^{b_{k}}\right\}
$$

isomorphic with $\mathfrak{F}$, and a subgroup

$$
\Omega_{G}=\left\{U_{1}^{a_{1}}, U_{2}^{a_{2}}, \ldots, U_{k}{ }^{a_{k}}\right\}
$$

isomorphic with 3 . These have no element in common (except the identity), and each generator of $\Omega_{F}$ commutes with each generator of $\Omega_{G}$. Hence the $2 k$ elements

$$
U_{r}^{a_{r}}, U_{r}^{b_{r}} \quad(r=1,2, \ldots, k)
$$

will generate the direct product $\Omega_{F} \times \Omega_{G}$, isomorphic with $\mathfrak{F} \times \nLeftarrow$. To verify that this subgroup $\Omega_{F} \times \Omega_{G}$ coincides with the whole group $\Omega$, we express the generators $U_{r}$ of the latter in terms of these particular powers, as follows. Since $\left(a_{r}, b_{r}\right)=1$, we can find positive or negative integers $\alpha_{r}$ and $\beta_{r}$ such that

then

$$
\alpha_{r} a_{r}+\beta_{r} b_{r}=1 ;
$$

$$
\left(U_{r}^{a_{r}}\right)^{\alpha_{r}}\left(U_{r}^{b_{r}}\right)^{\beta_{r}}=U_{r} \quad(r=1,2, \ldots, k) .
$$

Example. Take $\mathfrak{F}$ to be the $\mathfrak{D}_{2 n}$ defined by the generating relations

$$
R_{1}{ }^{n}=R_{2}{ }^{2}=\left(R_{1} R_{2}\right)^{2}=1,
$$

and $(5)$ the same dihedral group, but with the generating relations

$$
S_{1}{ }^{2}=S_{2}{ }^{n}=\left(S_{1} S_{2}\right)^{2}=1 .
$$

Then if $n$ is odd, the group $\Omega=\left\{U_{1}, U_{2}\right\}$ defined by

$$
\begin{aligned}
& U_{1}^{2 n}=U_{2}^{2 n}=\left(U_{1}^{2} U_{2}^{n}\right)^{2}=\left(U_{1}^{n} U_{2}^{2}\right)^{2}=1, \\
& U_{1}^{n} U_{2}^{n}=U_{2}^{n} U_{1}^{n}, \quad U_{1}{ }^{2} U_{2}{ }^{2}=U_{2}{ }^{2} U_{1}{ }^{2}
\end{aligned}
$$

will be isomorphic with the direct product $\mathfrak{D}_{2 n} \times \mathfrak{D}_{2 n}$.

Similarly, the direct product of two icosahedral groups,

and

$$
R_{1}{ }^{5}=R_{2}{ }^{3}=\left(R_{1} R_{2}\right)^{2}=1
$$

is

$$
S_{1}{ }^{3}=S_{2}{ }^{5}=\left(S_{1} S_{2}\right)^{2}=1,
$$

(cf. 2, p. 322).

$$
\begin{aligned}
& U_{1}^{15}=U_{2}^{15}=\left(U_{1}^{3} U_{2}^{5}\right)^{2}=\left(U_{1}^{5} U_{2}^{3}\right)^{2}=1, \\
& U_{1}^{5} U_{2}^{5}=U_{2}^{5} U_{1}^{5}, \quad U_{1}^{3} U_{2}^{3}=U_{2}^{3} U_{1}^{3}
\end{aligned}
$$

Remark. When $k=1$, this combination principle reduces to the well known fact that the direct product of two cyclic groups with relatively prime orders is again a cyclic group. Another special case, given by

$$
b_{2}=\ldots=b_{k}=1 \text {, }
$$

is Theorem II of Carmichael (1, p. 167). An extension of our principle to more than two "factors" is obvious. 
4. Application of the duplication principle to the construction of symmetrical graphs of degree three. Any group (5) generated by three involutory elements has a graph of degree three for its "Cayley colour group"; e.g., the Abelian group $\mathfrak{S}_{2} \times \mathfrak{S}_{2} \times \mathfrak{S}_{2}$ is represented by the vertices and edges of a cube $(7$, p. 38). If $\$$ admits an automorphism such that the three generators undergo a cyclic permutation, then the graph is symmetrical (6, p. 243). A great variety of symmetrical graphs can be derived from groups in this manner; the simplest that cannot is Petersen's graph (as R. M. Foster observed in a letter to the author).

A group (5) whose generators $T_{1}, T_{2}, T_{3}$ are involutory can be derived by applying the duplication principle to any finite group $\mathfrak{S}$ with $k=2$. Expressing $\mathfrak{S}$ in terms of three generators $S_{1}, S_{2}, S_{3}$ whose product is 1 , we see that an automorphism of (f) that cyclically permutes the $T$ 's arises from an automorphism of $\mathfrak{S}$ that cyclically permutes the $S$ 's. Accordingly, we say that a group $\mathfrak{S}$ with two generators, $S_{1}$ and $S_{2}$, fulfils the symmetry condition if it admits an automorphism such that the three elements $S_{1}, S_{2}$ and

$$
S_{3}=\left(S_{1} S_{2}\right)^{-1}=S_{2}^{-1} S_{1}^{-1}
$$

undergo a cyclic permutation.

Starting with any group $\mathfrak{S}$ of order $n$, that fulfils the symmetry condition, the duplication principle will yield a symmetrical graph of degree three having $2 n$ vertices and $3 n$ edges.

It should, however, be remarked that non-isomorphic groups of the same order can sometimes give rise to the same graph (as has been observed by Foster). For instance, Coxeter's graph $\{12\}+\{12 / 5\}$, formed by the 24 vertices and 36 edges of the hexagonal net $\{6,3\}_{2,2}$ on a torus (5, pp. 437440 ), arises from two distinct groups of order 12: the tetrahedral group $\mathfrak{I}_{12}$, defined by

4.1

$$
S_{1}^{3}=S_{2}^{3}=\left(S_{1}^{-1} S_{2}\right)^{2}=1 \text {, }
$$

and the Abelian group

or

$$
S_{1}^{2}=S_{2}^{2}=\left(S_{1} S_{2}\right)^{-2}, \quad S_{1} S_{2}=S_{2} S_{1}
$$

$$
S_{1}^{2}=S_{2}^{2}=S_{3}^{2}, \quad S_{1} S_{2} S_{3}=S_{3} S_{2} S_{1}=1,
$$

which is the direct product $\mathfrak{G}_{2} \times \mathfrak{G}_{6}$ or $\mathfrak{G}_{2} \times \mathfrak{E}_{2} \times \mathfrak{E}_{3}$.

It is clear that the graphs obtained by applying the duplication principle to suitable groups are always of the special kind which König calls "paar" (8, p. 170): the vertices of such a graph can be coloured alternately blue and red in such a manner that two vertices of the same colour are never joined by an edge. An important class of "paar" graphs are the "Levi graphs" of configurations (5, p. 413); e.g., the graph $\{12\}+\{12 / 5\}$, mentioned above, is the Levi graph for a configuration $12_{3}$. Although symmetrical graphs that are not "paar" cannot be obtained by the duplication principle, they may still sometimes be obtainable as "Cayley colour groups"; e.g., Foster's graph of 
girth 9 with 60 vertices $(11$, p. 459$)$ represents the icosahedral group generated by the three permutations

$$
\text { (1 2) (3 5), (1 3) (4 5), (1 4) (2 5). }
$$

Incidentally, the same icosahedral group can also be generated by the two elements

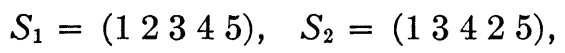

which satisfy the symmetry condition with $S_{3}=(14235)$. Applying to this group $\mathfrak{S}=\left\{S_{1}, S_{2}\right\}=\left\{S_{1}, S_{2}, S_{3}\right\}$ the duplication principle, we obtain the extended icosahedral group $\mathfrak{V}_{2} \times \mathfrak{S}$ generated by

$$
T_{1}=(12)(35)(67), \quad T_{2}=(13)(45)(67), \quad T_{3}=(14)(25)(67),
$$

and this yields a graph of girth 10 with 120 vertices (also found by Foster).

\section{Further examples of groups satisfying the symmetry condition.}

5.1. Abelian groups (as well as some others such as the above-mentioned tetrahedral group) yield graphs whose girth is 6 (or less). Indeed, the relation $S_{1} S_{2}^{-1} S_{1}^{-1} S_{2}=1$, which holds in an Abelian group $\mathfrak{S}=\left\{S_{1}, S_{2}\right\}$, corresponds in $(5)$ to the relation

$$
\left(T_{1} T_{2} T_{3}\right)^{2}=1 \text {, }
$$

which gives rise to hexagons in the graph.

In particular, the Abelian group

$$
S_{1}^{b}=S_{2}^{c}=S_{1}^{-c} S_{2}^{-b}, \quad S_{1} S_{2}=S_{2} S_{1}
$$

or

$$
S_{1}^{b}=S_{2}{ }^{c}, \quad S_{2}^{b}=S_{3}^{c}, \quad S_{3}^{b}=S_{1}^{c}, \quad S_{1} S_{2} S_{3}=S_{3} S_{2} S_{1}=1
$$

yields the graph of vertices and edges of the regular map $\{6,3\}_{b, c}$ on a torus (5, p. 421). This group, of order

$$
n=b^{2}+b c+c^{2},
$$

is $\mathfrak{S}_{d} \times \mathfrak{G}_{n / d}$, where $d=(b, c)$.

If $d=1$, it is simply the cyclic group $\mathfrak{S}_{n}$. Since

$$
(2 b+c)^{2}=4 n-3 c^{2},
$$

the possible values of $n$ are

$$
3,7,13,19,21,31, \ldots
$$

for all of which (except the first) -3 is a quadratic residue. Thus $\mathfrak{C}_{n}$ yields a graph with $2 n$ vertices whenever $n$ is an odd prime not of the form $6 m+5$, or a square-free product of such primes. In this case the congruence

$$
x^{2}+x+1 \equiv 0 \quad(\bmod n)
$$

or

$$
(2 x+1)^{2} \equiv-3
$$

$(\bmod 4 n)$ 
has a solution $x$, and the same graph $\{6,3\}_{b, c}$ arises from the same cyclic group $\mathfrak{C}_{n}$ in the form

Since

$$
S_{1}{ }^{n}=1, \quad S_{1}^{x}=S_{2} .
$$

$$
S_{1}^{x}=S_{2} \text { and } S_{2}^{x}=S_{1}^{x^{2}}=S_{1}^{-x-1}=S_{2}^{-1} S_{1}^{-1} \text {, }
$$

the symmetry condition is fulfilled by the automorphism which transforms each element of $\mathfrak{C}_{n}$ into its $x$ th power.

Setting $c=0$ in 5.11, we obtain the direct product $\mathfrak{E}_{b} \times \mathfrak{E}_{b}$, defined by

$$
S_{1}^{b}=S_{2}^{b}=1, \quad S_{1} S_{2}=S_{2} S_{1}
$$

or

$$
S_{1}^{b}=S_{2}^{b}=S_{3}^{b}=S_{1} S_{2} S_{3}=S_{3} S_{2} S_{1}=1
$$

(3, p. 97), which yields the graph $\{6,3\}_{b, 0}$ with $2 b^{2}$ vertices.

Another instance, given by $c=b$, is the $\mathfrak{S}_{b} \times \mathfrak{S}_{3 b}$ defined by

$$
S_{1}^{b}=S_{2}^{b}=S_{3}^{b}, \quad S_{1} S_{2} S_{3}=S_{3} S_{2} S_{1}=1,
$$

which yields the graph $\{6,3\}_{b, b}$ with $6 b^{2}$ vertices.

5.2. A non-Abelian group of order $3 b^{2}$, yielding the same graph $\{6,3\}_{b, b}$, is

or

$$
S_{1}{ }^{3}=S_{2}{ }^{3}=\left(S_{1} S_{2}\right)^{3}=\left(S_{1}{ }^{-1} S_{2}\right)^{b}=1
$$

$$
S_{1}{ }^{3}=S_{2}{ }^{3}=S_{3}{ }^{3}=S_{1} S_{2} S_{3}=\left(S_{1}{ }^{-1} S_{2}\right)^{b}=\left(S_{2}{ }^{-1} S_{3}\right)^{b}=\left(S_{3}{ }^{-1} S_{1}\right)^{b}=1
$$

(3, pp. 99-100). When $b=3$, these relations are equivalent to

$$
S_{1}{ }^{3}=S_{2}{ }^{3}=1, \quad S_{1}{ }^{-1} S_{2}{ }^{-1} S_{1} S_{2} . S_{i}=S_{i} . S_{1}{ }^{-1} S_{2}{ }^{-1} S_{1} S_{2} \quad(i=1,2)
$$

(1, p. 38, Ex. 26, 29) or

$5.21 \quad S_{1}{ }^{3}=S_{2}{ }^{3}=S_{3}{ }^{3}=S_{1} S_{2} S_{3}=1, \quad S_{2} S_{1} S_{3}=S_{3} S_{2} S_{1}=S_{1} S_{3} S_{2}$.

Thus another generalization is

$5.22 \quad S_{1}^{b}=S_{2}{ }^{b}=S_{3}{ }^{b}=S_{1} S_{2} S_{3}=1, \quad S_{2} S_{1} S_{3}=S_{3} S_{2} S_{1}=S_{1} S_{3} S_{2}$,

of order $\lambda b^{2}$, where $\lambda$ is the period of $S_{3} S_{2} S_{1}$, which is apparently $b$ or $\frac{1}{2} b$ according as $b$ is odd or even.

When $b=4$, this is a group $\mathfrak{S}_{32}$ of order 32 , defined by

$$
S_{1}{ }^{4}=S_{2}{ }^{4}=\left(S_{1} S_{2}\right)^{4}=1, \quad S_{1}{ }^{2} S_{2}=S_{2} S_{1}{ }^{2}, \quad S_{1} S_{2}{ }^{2}=S_{2}{ }^{2} S_{1},
$$

which gives rise to the author's symmetrical graph of girth 8 with 64 vertices (6, p. $244(3)$ ).

Extending 5.21 another way, we obtain

$$
S_{1}{ }^{3}=S_{2}{ }^{3}=S_{3}{ }^{3}, S_{1} S_{2} S_{3}=1, S_{2} S_{1} S_{3}=S_{3} S_{2} S_{1}=S_{1} S_{3} S_{2}=Z, Z^{3}=1,
$$

of order 81 , and

$$
S_{1}{ }^{9}=S_{2}{ }^{9}=S_{3}{ }^{9}=S_{1} S_{2} S_{3}=1, S_{2} S_{1} S_{3}=S_{3} S_{2} S_{1}=S_{1} S_{3} S_{2}=Z, Z^{3}=1,
$$


of order 243 . The former yields a new symmetrical graph of girth 12 with 162 vertices: The simplest known graph of girth 12 .

5.3. The quaternion group $\mathfrak{Q}_{8}$, defined by

or

$$
S_{1}^{2}=S_{2}^{2}=\left(S_{1} S_{2}\right)^{2}
$$

$$
S_{1}{ }^{2}=S_{2}{ }^{2}=S_{3}{ }^{2}, \quad S_{1} S_{2} S_{3}=1
$$

gives rise to a graph of girth 6 with 16 vertices, the $\{8\}+\{8 / 3\}$ of Coxeter (5, p. 430).

Applying the combination principle to this and the $\mathfrak{C}_{3}$

$$
R_{1}{ }^{3}=1, \quad R_{1}=R_{2},
$$

we obtain the direct product $\mathfrak{C}_{3} \times \mathfrak{\Omega}_{8}$ in the form

$$
\begin{aligned}
& U_{1}{ }^{2}=1, \quad U_{1}{ }^{4}=U_{2}{ }^{4}, \\
& U_{1}{ }^{6}=U_{2}{ }^{6}=\left(U_{1}^{3} U_{2}^{3}\right)^{2}, \\
& U_{1}^{3} U_{2}{ }^{4}=U_{2}^{4} U_{1}^{3}, \quad U_{1}{ }^{4} U_{2}^{3}=U_{2}^{3} U_{1}^{4},
\end{aligned}
$$

which easily reduces to

or

$$
U_{1}^{2}=U_{2}^{2}=\left(U_{1} U_{2}\right)^{-2}
$$

$$
U_{1}^{-2}=U_{2}^{-2}=U_{3}^{2}=U_{1} U_{2} U_{3}
$$

the $\langle-2,-2,2\rangle$ of Coxeter (4, pp. 367, 377). The duplication principle gives rise to a symmetrical graph of girth 8 , with 48 vertices, found by Foster.

5.4. Applying the combination principle to the tetrahedral group $\mathfrak{I}_{12}$ and the four-group $\mathfrak{C}_{2} \times \mathfrak{C}_{2}$, we obtain the group

$$
S_{1}{ }^{6}=S_{2}{ }^{6}=S_{3}{ }^{6}=S_{1} S_{2} S_{3}=\left(S_{1}{ }^{-1} S_{2}\right)^{2}=\left(S_{2}{ }^{-1} S_{3}\right)^{2}=\left(S_{3}{ }^{-1} S_{1}\right)^{2}=1,
$$

of order 48 , which can be generated by permutations

$$
\text { (1 } 23 \text { 3)(5 6)(7 8), (1 } 34)(78)(90), \quad(142)(90)(56),
$$

and yields a graph of girth 8 with 96 vertices. Similarly

$$
S_{1}{ }^{12}=S_{2}{ }^{12}=S_{3}{ }^{12}=S_{1} S_{2} S_{3}=\left(S_{1}{ }^{-1} S_{2}\right)^{2}=\left(S_{2}{ }^{-1} S_{3}\right)^{2}=\left(S_{3}{ }^{-1} S_{1}\right)^{2}=1,
$$

of order 96 (3, p. 101, footnote), yields a new graph of girth 8 with 192 vertices.

5.5. The group

$$
\begin{aligned}
& 5.51 \quad S_{1}{ }^{2 n}=S_{2}{ }^{2 n}=\left(S_{1} S_{2}\right)^{2 n}=1, \quad S_{1}{ }^{2} S_{2} S_{1}{ }^{2}=S_{2}, \quad S_{2}{ }^{2} S_{1} S_{2}{ }^{2}=S_{1} \\
& \text { or } \\
& S_{1}{ }^{2 n}=S_{2}{ }^{2 n}=S_{3}{ }^{2 n}=S_{1} S_{2} S_{3}=1, \quad S_{1}{ }^{2} S_{2} S_{1}{ }^{2}=S_{2}, S_{2}{ }^{2} S_{3} S_{2}{ }^{2}=S_{3}, \quad S_{3}{ }^{2} S_{1} S_{3}{ }^{2}=S_{1}, \\
& \text { of order } 4 n^{3} \text {, yields a graph with } 8 n^{3} \text { vertices, which has girth } 10 \text { if } n \geqslant 3 .
\end{aligned}
$$


Thus a graph of girth 10 with 216 vertices comes from the group of order 108 generated by

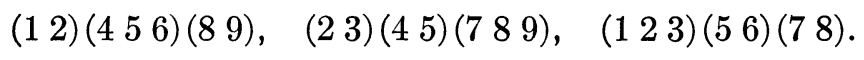

The above relations are easily seen to imply

$$
S_{1}{ }^{2} S_{2}{ }^{2}=S_{2}{ }^{2} S_{1}{ }^{2}, \quad S_{2}{ }^{2} S_{3}{ }^{2}=S_{3}{ }^{2} S_{2}{ }^{2}, \quad S_{3}{ }^{2} S_{1}{ }^{2}=S_{1}{ }^{2} S_{3}{ }^{2},
$$

so that the subgroup $\left\{S_{1}{ }^{2}, S_{2}{ }^{2}, S_{3}{ }^{2}\right\}$, of index 4 , is simply $\mathfrak{E}_{n} \times \mathfrak{G}_{n} \times \mathfrak{C}_{n}$. If $n$ is even, this subgroup has a factor group of order $\frac{1}{2} n^{3}$, given by inserting the extra relation

$$
S_{1}{ }^{n} S_{2}{ }^{n} S_{3}{ }^{n}=1 \text { or }\left(S_{1}{ }^{2} S_{2}{ }^{2} S_{3}{ }^{2}\right)^{\frac{1}{2} n}=1 \text { or }\left(S_{3} S_{2} S_{1}\right)^{\frac{1}{2} n}=1 .
$$

Hence the whole group has a factor group of order $2 n^{3}$ (when $n$ is even), given by inserting the extra relation

$$
\left(S_{1}^{-1} S_{2}^{-1} S_{1} S_{2}\right)^{\frac{1}{2} n}=1 \text { or }\left(S_{3} S_{2} S_{1}\right)^{\frac{1}{2} n}=1 .
$$

This yields a graph with $4 n^{3}$ vertices ( $n$ even), whose girth is 10 if $n \geqslant 4$. Thus a graph of girth 10 with 256 vertices comes from the group of order 128 defined by

$$
S_{1}^{8}=S_{2}^{8}=\left(S_{1} S_{2}\right)^{8}=\left(S_{1}^{-1} S_{2}^{-1} S_{1} S_{2}\right)^{2}=1, S_{1}{ }^{2} S_{2} S_{1}{ }^{2}=S_{2}, S_{2}{ }^{2} S_{1} S_{2}{ }^{2}=S_{1} .
$$

5.6. Similarly, the group

$$
5.61 \quad S_{1}{ }^{2 n}=S_{2}{ }^{2 n}=\left(S_{1} S_{2}\right)^{2 n}=1, \quad S_{1}{ }^{2} S_{2}=S_{2} S_{1}{ }^{2}, \quad S_{1} S_{2}{ }^{2}=S_{2}{ }^{2} S_{1}
$$

or

$S_{1}{ }^{2 n}=S_{2}{ }^{2 n}=S_{3}{ }^{2 n}=S_{1} S_{2} S_{3}=1, S_{1}{ }^{2} S_{2}=S_{2} S_{1}{ }^{2}, S_{2}{ }^{2} S_{3}=S_{3} S_{2}{ }^{2}, S_{3}{ }^{2} S_{1}=S_{1} S_{3}{ }^{2}$, of order $4 n^{3}$, yields a graph with $8 n^{3}$ vertices whose girth is 10 if $n \geqslant 3$; and if $n$ is even, it has a factor group of order $2 n^{3}$, given by inserting the extra relation

$$
\left(S_{1}^{-1} S_{2}^{-1} S_{1} S_{2}\right)^{\frac{1}{2} n}=1 \text { or }\left(S_{3} S_{2} S_{1}\right)^{\frac{1}{2} n}=1,
$$

which yields a graph with $4 n^{3}$ vertices.

When $n=2,5.51$ and 5.61 both reduce to 5.23 . But when $n>2$, the two graphs are distinct, as R. M. Foster found by examining them in detail.

5.7. A graph of girth 12 , with 288 vertices, is yielded by the group

$$
\begin{aligned}
S_{1}{ }^{6}=S_{2}{ }^{6}=S_{3}{ }^{6}=S_{1} S_{2} S_{3}=\left(S_{3} S_{2} S_{1}\right)^{2}=S_{3}{ }^{2} S_{2}{ }^{2} S_{1}{ }^{2}=1, \\
S_{1}{ }^{3} S_{2}=S_{2} S_{1}{ }^{3}, \quad S_{2}{ }^{3} S_{3}=S_{3} S_{2}{ }^{3}, \quad S_{3}{ }^{3} S_{1}=S_{1} S_{3}{ }^{3}
\end{aligned}
$$

of order 144, which is a subgroup of $\mathfrak{S}_{6} \times \mathfrak{S}_{6} \times \mathfrak{I}_{12}$.

5.8. A graph of girth 8 , with 336 vertices, results from the well-known simple group of order 168 in the form

$$
S_{1}{ }^{4}=S_{2}{ }^{4}=\left(S_{1}{ }^{-1} S_{2}\right)^{3}=\left(S_{1}{ }^{2} S_{2}\right)^{3}=1
$$

or 


$$
S_{1}{ }^{4}=S_{2}{ }^{4}=S_{3}{ }^{4}=S_{1} S_{2} S_{3}=\left(S_{1}{ }^{-1} S_{2}\right)^{3}=\left(S_{2}{ }^{-1} S_{3}\right)^{3}=\left(S_{3}{ }^{-1} S_{1}\right)^{3}=1
$$

(3, p. 96). As permutations of degree seven, we have

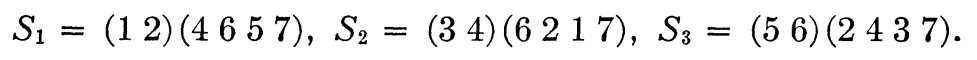

5.9. The author's one-regular graph of girth 12 , with 432 vertices (6, p. 246), comes from Hesse's collineation group of order 216 (9, p. 239) in the form

$S_{1}{ }^{6}=S_{2}{ }^{6}=S_{3}{ }^{6}=S_{1} S_{2} S_{3}=1, S_{1}{ }^{2} S_{2} S_{1}{ }^{2}=S_{2}{ }^{2}, S_{2}{ }^{2} S_{3} S_{2}{ }^{2}=S_{3}{ }^{2}, S_{3}{ }^{2} S_{1} S_{3}{ }^{2}=S_{1}{ }^{2}$.

As permutations of degree 9 , we have

$$
S_{1}=(48)(162395), \quad S_{2}=(59)(341276), \quad S_{3}=(67)(253184) \text {. }
$$

6. Concluding remarks. In each of the foregoing examples the duplication principle would allow us to indicate also generating relations for a group (5) whose order is twice that of the given group $\mathfrak{S}_{\mathfrak{S}}$. For instance, the group 5.22 yields

$$
\begin{aligned}
T_{1}{ }^{2}=T_{2}{ }^{2}=T_{3}{ }^{2}=\left(T_{2} T_{3}\right)^{b}=\left(T_{3} T_{1}\right)^{b}=\left(T_{1} T_{2}\right)^{b} & =1, \\
\left(T_{1} T_{2} T_{3}\right)^{2} & =\left(T_{2} T_{3} T_{1}\right)^{2}=\left(T_{3} T_{1} T_{2}\right)^{2},
\end{aligned}
$$

of order $2 \lambda b^{2}$ (where $\lambda=b$ or $\frac{1}{2} b$ according as $b$ is odd or even).

Again, 5.51 and 5.61 yield

$$
T_{1}^{2}=T_{2}^{2}=T_{3}^{2}=\left(T_{2} T_{3}\right)^{2 n}=\left(T_{3} T_{1}\right)^{2 n}=\left(T_{1} T_{2}\right)^{2 n}=1
$$

with the extra relations

and

$$
T_{1} T_{2} T_{1} T_{3} T_{1}=T_{2} T_{3} T_{2} T_{1} T_{2}=T_{3} T_{1} T_{3} T_{2} T_{3}
$$

$$
\left[T_{1}\left(T_{2} T_{3}\right)^{2}\right]^{2}=\left[T_{2}\left(T_{3} T_{1}\right)^{2}\right]^{2}=\left[T_{3}\left(T_{1} T_{2}\right)^{2}\right]^{2}=1,
$$

respectively. When $n=3$, the former has a factor group of order 108, given by the extra relation $\left(T_{1} T_{2} T_{3}\right)^{3}=1$; this yields Foster's graph of girth 9 with 108 vertices.

If the conditions for the combination principle are fulfilled, its application yields further symmetrical graphs corresponding to such direct products as

$$
\begin{array}{ccc}
\mathfrak{C}_{7} \times \mathfrak{S}_{8}, & \left(\mathfrak{S}_{3} \times \mathfrak{C}_{3}\right) \times \mathfrak{I}_{8}, & \mathfrak{S}_{7} \times \mathfrak{I}_{12}, \\
\mathfrak{S}_{3} \times \mathfrak{S}_{32}, & \mathfrak{I}_{8} \times \mathfrak{I}_{12}, & \mathfrak{D}_{8} \times \mathfrak{S}_{13},
\end{array}
$$

etc. When generating relations for each "factor" are known, the combination principle yields also generating relations for the direct product, as in $\$ 5.3$ for $\mathfrak{S}_{3} \times \mathfrak{Q}_{8}$, and in $\$ 5.4$ for $\left(\mathfrak{S}_{2} \times \mathfrak{G}_{2}\right) \times \mathfrak{I}_{12}$. 


\section{REFERENCES}

1. R. D. Carmichael, Introduction to the theory of groups of finite order (Boston), 1937.

2. H. S. M. Coxeter, Abstract definitions for the symmetry groups of the regular polytopes, in terms of two generators. Part II: The rotation groups, Proc. Camb. Philos. Soc., 33 (1937), 315-324.

3. The abstract groups $G^{m, n, p}$, Trans. Amer. Math. Soc., 45 (1939), 73-150.

4. - The binary polyhedral groups, and other generalizations of the quaternion group, Duke Math. J., 7 (1940), 367-379.

5. - Self-dual configurations and regular graphs, Bull. Amer. Math. Soc., 56 (1950), 413-455.

6. R. Frucht, $A$ one-regular graph of degree three, Can. J. Math., 4 (1952), 240-247.

7. A. B. Kempe, $A$ memoir on the theory of mathematical form, Phil. Trans. Royal Soc. A, $17 y$ (1886), 1-70.

8. D. König, Theorie der endlichen und unendlichen Graphen (Leipzig, 1936).

9. G. A. Miller, H. F. Blichfeldt, and L. E. Dickson, Theory and applications of finite groups (New York, 1916).

10. W. Threlfall, Gruppenbilder, Abh. Math.-Phys. Klasse Sächs. Akad. Wiss., 41.6 (1932), 1-59.

11. W. T. Tutte, $A$ family of cubical graphs, Proc. Cambridge Philos. Soc., 43 (1947), 459-474.

Technical University "Santa Maria"

Valparaiso, Chile 